The amount of gain, its onset at a drift field high enough for the electron drift velocity to equal the elastic wave velocity, and the observed decrease in gain upon changing the resistivity from the optimum value, are all similar to the case of bulk wave amplification. It should be noted that in these experiments the drift field existed only between the transducers, whereas the illumination was uniform both between and beneath the transducers, producing some constant attenuation at the transducers. A higher gain per unit length would be observed if the illumination or crystal conductivity were altered so as to eliminate this loss beneath the transducers.

Surface-wave propagation has been observed in CdS crystals having their $c$ axes either normal or parallel to the surface plane. For both orientations, a surface-wave velocity of $(1.73 \pm 0.05) \times 10^{5} \mathrm{~cm} / \mathrm{sec}$ was inferred from the period of the transducer electrodes $\left(2.20 \times 10^{-2} \mathrm{~cm}\right)$ and the measured frequency which maximized the received pulse amplitude. This velocity agrees well with the value $1.70 \times$ $10^{5} \mathrm{~cm} / \mathrm{sec}$ calculated for CdS from an expression derived by Stoneley ${ }^{3}$ which is applicable to the firstmentioned orientation.

The velocity of surface waves in CdS is only slightly smaller than the velocity of transverse bulk waves. That we have indeed dealt here with surface waves rather than bulk transverse waves is shown by the following: (a) velocity measurements in experiments with crystalline quartz (in which the velocities of bulk and surface waves are well separated) showed that the electrodes function as surface wave transducers; (b) on quartz, excellent transmission was observed from one electrode transducer to a metallic comb surface-wave transducer as described by Viktorov, ${ }^{4}$ and Arzt and Dransfeld,; ${ }^{5}$ (c) a drop of acetone or alcohol on the CdS surface produced apparently complete absorption of the elastic pulse; (d) probing the CdS crystal with a narrow beam of light directed parallel to the plane surface produced a variable attenuation of the received pulse which showed that the wave amplitude was high near the surface and that it was negligible at large depths.

This work was supported in part by the U. S. Army Research Office-Durham, under Grant DA-ARO-D-31-124-G678.

\footnotetext{
${ }^{1}$ H. Kolsky, Stress Waves in Solids (Clarendon Press, Oxford, 1953).

${ }^{2}$ D. L. White, J. Appl. Phys. 33, 2547 (1962).

${ }^{3}$ R. Stoneley, Geophys. Suppl. to Monthly Notes, Roy. Astron. Soc. 5, $343(1949)$.

${ }^{4}$ I. A. Viktorov, Sov. Phys. Acoustics 7, 236 (1962).

${ }^{5}$ R. M. Arzt and K. Dransfeld, Appl. Phys. Letters 7, 156 (1965).
}

\title{
TWO-BEAM INTERFEROMETRY BY SUCCESSIVE RECORDING OF INTENSITIES IN A SINGLE HOLOGRAM
}

(image synthesis; complex addition of wave fronts; T/E)

Heretofore in optics, two-beam interferograms of a wave front scattered by an object have been generally recorded by comparing the scattered wave front (e.g. reflected, transmitted or diffracted by an object) to a wave front simultaneously reflected from a reference mirror, for example in a beam-splitting interferometer, such as that introduced by Michelson and others. More generally, two different wave fronts can thus be added with the aid of beam splitters or equivalent arrangements. These interferometers permit the complex amplitudes of the two wave fronts to be added, provided that the two wave fronts are made to interfere simultaneously.
George W. Stroke and Antoine E. Labeyrie The University of Michigan Ann Arbor, Michigan

(Received 17 November 1965; in final form 3 January 1966)

In this Letter we show that two-beam interferograms can be obtained by wave-front reconstruction from a hologram, which was recorded by having a coherent background successively interfere in the same latent image, first with the wave "transmitted" through the object and next with the wave incident on the object, or vice versa. Our method is based on a method which we may call a method of "holographic intensity interferometry," first described by Gabor and Stroke et al., ${ }^{1}$ who showed that successive addition of intensities in a hologram could be made equivalent to a simultaneous addition of complex amplitudes in an interferometer. More pre- 
cisely we showed in our ref. 1 that complex amplitudes could be added, of not just two but indeed of several wave fronts, by making each of the wave fronts first interfere in succession (in the latent photographic image) with a same coherent-background reference wave, thus adding in succession the intensities of the individual partial holograms, so as to retrievably store in the resultant hologram the complex addition of the several wave fronts.

As an example, Gabor and Stroke et al. ${ }^{1}$ considered an "image synthesis" method, in which the complex amplitudes of two images were holographically subtracted according to this principle.

The application of our method of holographic intensity interferometry ${ }^{1}$ to two-beam interferometry, which we describe here, has also permitted us to further clarify a particular form of linearity in holography, according to which successive holographic superposition of complex amplitudes in wave fronts can be made to be equivalent to a simultaneous superposition in "classical" interferometry.

As a model we discuss the holographic recording and reconstruction arrangements shown in Fig. 1a and Fig. Ib. Consider first the recording process which is, itself, formed of two successive steps (Fig. 1a). In the first step, the object $\mathrm{O}$ is not in the beam originating from $R$. Let $a_{S}(x)$ and $a_{R}(x)$ be the complex amplitude distributions produced on the hologram-recording plate by the waves originat-
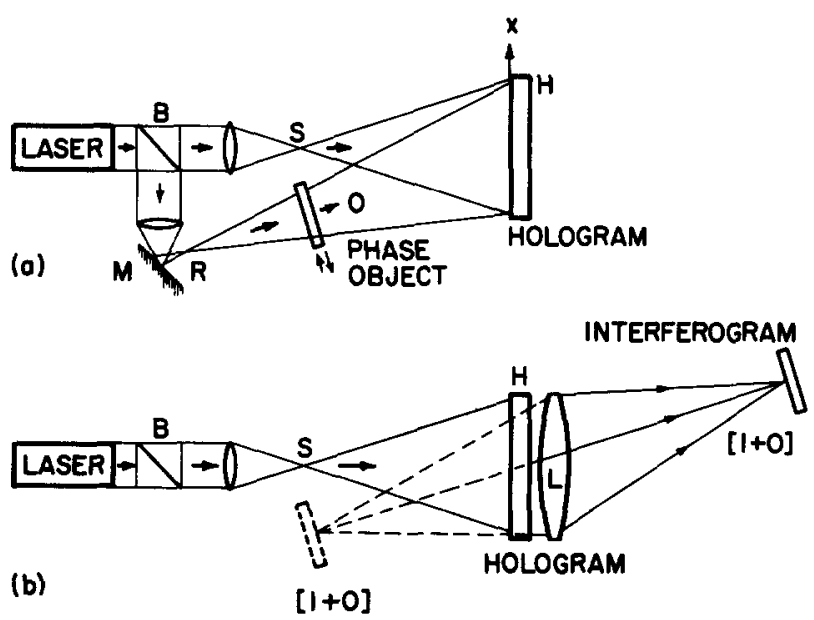

Fig. 1. (a) Recording of hologram and (b) reconstruction of two-beam interferogram in holographic intensity interferometry. Partial intensities in the same latent photographic image in the single hologram are recorded by making the beam from $\boldsymbol{R}$ successively interfere with the coherent background reference beam from $S$, first with the object in the beam, and next without the object in the beam, or vice versa. Equivalent arrangements using reflection or diffraction from objects, or gratings, for example, are readily conceivable. ing from $S$ and from $R$, where $a_{R}(x)$ is the amplitude produced on the hologram without the object $O$ in the beam. The corresponding partial intensity recorded in the latent photographic image is

$$
I_{1}(x)=a_{S}^{2}+a_{R}^{2}+a_{S} a_{R}^{*}+a_{S}^{*} a_{R} \text {. }
$$

In the second step of the recording process, the object $\mathrm{O}$ is placed in the beam originating from $R$ (Fig. 1a), and this without disturbing the hologramrecording plate from its first-step position. With the object $\mathrm{O}$ thus placed in the beam, the partial in tensity $I_{2}(x)$ added to the "latent" partial intensity $I_{1}(x)$ is

$$
I_{2}(x)=a_{S}^{2}+a_{o}^{2}+a_{S} a_{o}^{*}+a_{S}^{*} a_{o},
$$

where $a_{0}(x)$ is the complex amplitude produced in the $x$ plane by the wave $A_{0}$, where $A_{0}$ was produced by transmission through the object $\mathrm{O}$ (of which the complex-amplitude transmission is equal to 0 ) of the wave $A_{R}$ originating from $R$ (see Fig. la). We have $A_{o}=A_{R} \mathrm{O}$. The total exposure of the hologram in the two-step "double-exposure" process" is

$$
\begin{aligned}
I=I_{1}+I_{2}=\left(2 a_{S}^{2}+a_{R}^{2}+a_{0}^{2}\right)+ & a_{S}\left(a_{R}^{*}+a_{0}^{*}\right) \\
& +a_{S}^{*}\left(a_{R}+a_{0}\right) .
\end{aligned}
$$

The reconstruction process is illustrated in Fig. $1 \mathrm{~b}$. Because of the usual linearity conditions characteristic of holography, ${ }^{2,7}$ illumination of the hologram (as in Fig. 1b) by the wave $a_{S}(x)$ originating from $S$ reconstructs the wave fronts corresponding to the preceding equation, and produces, in particular, two aerial images, respectively $\left(A_{R}^{*}+A_{o}^{*}\right)$ and $\left(A_{R}+A_{0}\right)=A_{R}(1+\mathrm{O})$. The image $A_{R}(1+\mathrm{O})$ is nothing but a two-beam interferogram formed between the reference wave $A_{R}$ and the wave $A_{R} \mathrm{O}$ (equal to the complex amplitude transmission of the phase object $\mathrm{O}$, when illuminated by the wave $A_{R}$ ). In other words, the reconstructed image $A_{R}(1+\mathrm{O})$ displays a two-beam "equal-thickness" interference fringe system characteristic of the phase transmission of the object $O$.

An example of a two-beam interferogram of a phase object obtained according to the scheme which we describe is shown in Fig. 2. [The phase object was produced by photographing the word "phase" (20 mm long) and the symbol " $\phi$ " on a Kodak $649 \mathrm{~F}$ microflat plate, and by bleaching the developed and fixed plate in Kodak chromium intensifier, so as to obtain a practically "pure" phase 
object (without amplitude contrast), such as that which might occur in acoustical applications, or in image "coding," or in microscopy, for example. (The phase object is the same object as that used in the work on reconstruction of phase objects by holography by Gabor and Stroke et al. $\left.{ }^{3}\right)$ ]

It may be of interest to note that the preceding scheme of holographic interferometry produces two-beam interferograms which are not affected by

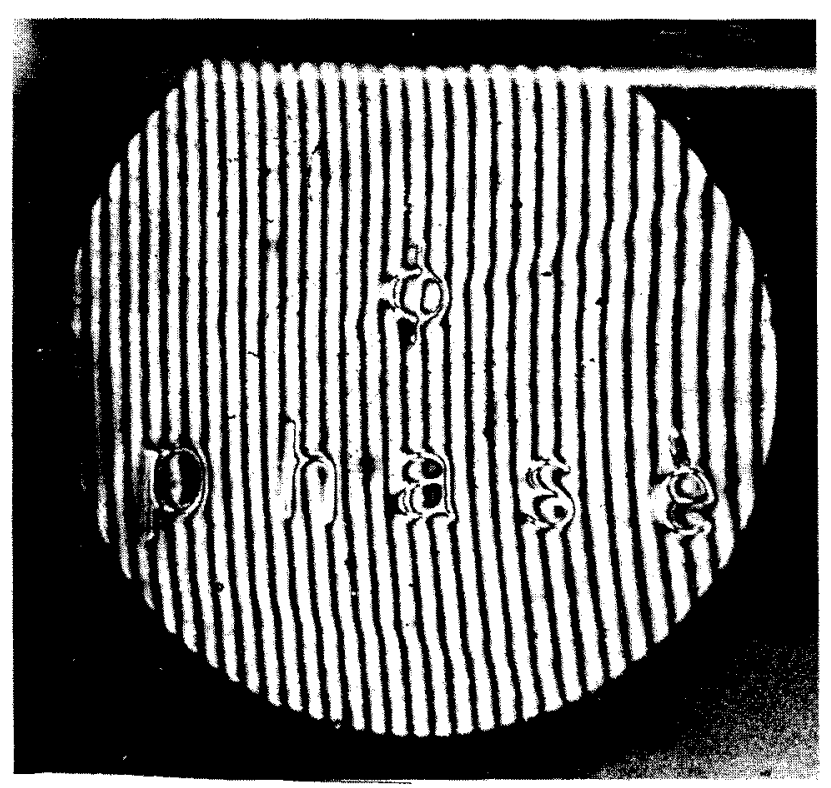

Fig. 2. Two-beam interferogram of a phase object (about 20 $\mathrm{mm}$ long) ${ }^{3}$ reconstructed as in Fig. $1 \mathrm{~b}$ from a hologram recorded according to Fig. 1a (see text). aberrations of the interferometer elements. We may also note that some analogies could be made between the relation of classical interferometry and our method of "holographic intensity interferometry," on the one hand, and on the other, the relation of two-slit (e.g. stellar) interferometry and the method of "intensity interferometry" first described by Hanbury-Brown and Twiss ${ }^{4}$ in 1954 (see also our ref. 7). Analogies of our method of holographic interferometry with some other recently described methods, ${ }^{5,6}$ also including extensions of our principle of multiple-exposed holograms (as first described by Gabor and Stroke et al. in our ref. 1) could also be readily made.

We wish to acknowledge fruitful conversations with Professor D. Gabor and with D. Brumm, A. Funkhouser, and R. C. Restrick, and the generous help of the Office of Naval Research in supporting part of this work.

'D. Gabor and G. W. Stroke, R. Restrick, A. Funkhouser, and D. Brumm, Phys. Letters 18, 116 (1965).

${ }^{2}$ D. Gabor, Proc. Roy. Soc. (London) B64, $449(1951)$.

${ }^{3}$ D. Gabor and G. W. Stroke, D. Brumm, A. Funkhouser and A. Labeyrie, Nature 208, 1159 (1965).

${ }^{4}$ R. Hanbury-Brown and R. Q. Twiss, Phil. Mag. (7) 45, 663 (1954).

${ }^{5}$ e.g. R. J. Collier, E. T. Doherty, and K. S. Pennington, Appl. Phys. Letters 7, 223 (1965).

${ }^{6}$ e.g. R. E. Brooks, L. O. Heflinger, and R. F. Wuerker, Appl. Phys. Letters 7, 248 (1965).

${ }^{7}$ For a general background, see e.g. G. W. Stroke, An Introduction to Coherent Optics and Holography (Academic Press, New York and London, 1965) (Library of Congress Catalog Card Number: $65-28633$ ).

\section{GHOST IMAGING BY HOLOGRAMS FORMED IN THE NEAR FIELD}

(no auxiliary reference beam; $\mathrm{E} / \mathrm{T}$ )

The hologram-generated ghost images predicted by Van Heerden ${ }^{1}$ have recently been observed by Stroke et al. ${ }^{2}$ for the case where a subject transparency is illuminated by collimated laser light and by Pennington and Collier ${ }^{3}$ for the case where the transparency is illuminated by diffuse laser light. The present Letter reports ghost imaging of solid objects, without the use of Fourier transforming lenses, so that the hologram plate is in the near field of the subject scene.
R.J. Collier and K. S. Pennington Bell Telephone Laboratories, Incorporated Murray Hill, New Jersey (Received 8 December 1965 )

The hologram is formed by diffuse reflection of 6328 - $\AA$ laser light from two rectangular metal bars as shown in Fig. 1. The vertical bar was about $1 \mathrm{in}$. closer to the hologram plate than the horizontal one. A bright point source of phase-related laser light was included as part of the subject scene to aid in reregistering the developed hologram in the exact position it had during formation. Providing that the hologram is reregistered, that the subject illumination has remained constant and the point source 